\title{
PipaBot: um canal de comunicação para o PIPA UFRJ
}

\author{
Lucas S. de Paula ${ }^{\mathrm{a}}$, Taisa G. Gonçalves ${ }^{\mathrm{b}}$, Thatiana V. R. B. Fernandes ${ }^{\mathrm{c}}$, Guilherme H. Travassos ${ }^{\mathrm{b}}$ \\ $\mathrm{ECI} / \mathrm{POLI}^{\mathrm{a}}, \mathrm{PESC} / \mathrm{COPPE}^{\mathrm{b}}, \mathrm{IESC}_{\mathrm{CSS}}^{\mathrm{c}}$ \\ Universidade Federal do Rio de Janeiro (UFRJ) \\ Rio de Janeiro, Brasil \\ lucasdepaula@poli.ufrj.br, taisa@cos.ufrj.br, thatianafernandes@iesc.ufrj.br, ght@cos.ufrj.br
}

\begin{abstract}
Chatbots make use of natural language processing to establish dialogues with people. They can be used as channels that facilitate communication between humans and entities (companies, social projects, research projects, hospitals, schools/universities, etc.). The PipaBot is a chatbot that was built to facilitate the communication between the mothers (expected two thousand participants) and researchers from the Childhood and Environmental Pollutants Project (PIPA-UFRJ). It was built following an incremental and iterative way and used the Botpress framework. The chatbot is available in two versions: Facebook Messenger and PIPA-UFRJ website. The actions provided by PipaBot are (i) registration of a project participant; (ii) information about the project, medical consultations, and result of medical exams, among others. The PipaBot was evaluated following the Technological Acceptance Model (TAM) with 13 participants, and the initial results provided evidence of user satisfaction regarding its acceptance.
\end{abstract}

\section{KEYWORDS}

Chatbot, intelligence artificial, software engineering, bootpress framework, Technological Acceptance Model

\section{Introdução}

Desde seu marco inicial em 1950 com o artigo científico "Computing Machinery and Intelligence" a Inteligência Artificial (IA) vem sendo aplicada à diferentes áreas da ciência e da indústria (pesquisas médicas, educação, agricultura, robótica, esporte, assistência jurídica, varejo, manufatura, governo, atendimento ao cliente, dentre outros) [7][9].

Segundo Lu [9], a IA é representada por qualquer teoria, método e técnica que ajuda as máquinas (especialmente os computadores) a analisar, simular e explorar o processo e o comportamento do pensamento humano. A IA possui limitações, e uma delas é que ela aprende com os dados. O conhecimento não é incorporado como acontece com os seres humanos. Em particular, a indústria vem utilizando os sistemas de perguntas e respostas (chatbots) que aplicam conceitos da IA.

In: XVIII Workshop de Ferramentas e Aplicações (WFA 2019), Rio de Janeiro, Brasil. Anais Estendidos do Simpósio Brasileiro de Sistemas Multimídia e Web (WebMedia). Porto Alegre: Sociedade Brasileira de Computação, 2019.

(C)2019 SBC - Sociedade Brasileira de Computação.

ISSN: $2596-1683$
Um Chatbot é um sistema de software que tem como objetivo simular um ser humano nas conversas com pessoas, fazendo uso do processamento de linguagem natural [1]. Chatbots podem ser integrados à websites [1][7], aplicativos como o Facebook Messenger, Telegram [1] e WhatsApp; ou mesmo integrados à sistemas IoT (Internet das Coisas) genéricos [8] ou nas áreas de previsão do tempo [4], sensoriamento [6], entre outros.

Considerando a utilização de chatbots em diferentes sistemas de software, o objetivo deste trabalho é apresentar o PipaBot, o chatbot criado para o Projeto Infância e Poluentes Ambientais (PIPA UFRJ). Este projeto acompanha mães e seus bebês com o objetivo de avaliar os efeitos de poluentes ambientais na fase de gestação até o início da infância (quatro anos).

O PipaBot foi desenvolvido para ser um canal de comunicação entre os responsáveis do projeto e as mães. Tendo como base o framework Botpress que integra conceitos de IA, o chatbot foi desenvolvido de forma iterativa e integrado ao Messenger do Facebook e ao website do PIPA UFRJ. Ele foi avaliado usando o modelo de aceitação da tecnologia (TAM Tecnological Acceptance Model) e é capaz de dialogar com os usuários a respeito de dúvidas e informações sobre o PIPA UFRJ, sobre consultas, resultados de exames médicos, entre outros.

Além desta introdução, este artigo apresenta três seções. A seção 2 apresenta os conceitos fundamentais de um chatbot e discute algumas soluções de chatbot encontradas na literatura. A seção 3 apresenta o processo de desenvolvimento do PipaBot. Por fim, a seção 4 apresenta a conclusão e os trabalhos futuros.

\section{Fundamentação teórica}

Os chatbots são agentes inteligentes computadorizados que simulam conversas com pessoas usando processamento de linguagem natural (PLN) e outras técnicas [7]. Muitas vezes são nomeados como assistentes virtuais, agentes virtuais ou, simplesmente, bot. O primeiro software para PLN (chamado ELIZA) foi criado por Joseph Weizenbaum [13] no laboratório de IA do MIT (Massachusetts Institute of Technology). Outro exemplo de chatbot é A.L.I.C.E. (Artificial Linguistic Internet Computer Entity), criado por Richard S. Wallace em 1995 [12].

Chatbots podem ser classificados em dois tipos principais: baseado em regras e baseado em IA [7]. Chatbots baseados em regras possuem fluxos de navegação definidos e palavras-chave parametrizadas (respostas automáticas) de acordo com o domínio de aplicação. No caso de uso de técnicas de IA, o chatbot é mais versátil e complexo. Ele é treinado através de processamento de 
linguagem natural para identificar contextos de conversas. As intenções do usuário são identificadas e a resposta mais adequada é enviada. O chatbot aprende as respostas com o tempo, ou seja, quanto mais ele é utilizado, mais "inteligente" ele fica.

Uma vantagem dos chatbots é que eles facilitam as interações, principalmente as repetitivas (no caso de atendimento ao cliente), além de motivar o usuário a participar da conversação [2]. Outras vantagens são: disponibilidade $24 \mathrm{~h}$ por dia, 7 dias por semana; ajuda na automatização dos processos; além de possibilitar múltiplos canais de comunicação.

O mercado oferece algumas ferramentas para o desenvolvimento de chatbots tais como: Blip (https://blip.ai/), Microsoft BotPlataform (https://dev.botframework.com/), Botpress (https://botpress.io/) e Pandorabots (https://home.pandorabots.com/home.html). Por exemplo, Blip é uma plataforma que permite estruturar um chatbot e colocá-lo em funcionamento em poucos passos. O desenvolvedor deve definir o fluxo, criar o conteúdo da conversa e publicar o seu chatbot. A versão standard é gratuita e a versão business demanda contratação mensal.

A literatura técnica apresenta algumas soluções de chatbots no domínio da saúde. AGEbot [7] é um robô de conversação desenvolvido para responder dúvidas sobre a área da epilepsia. Ele foi desenvolvido com base no Pandorabots (https://home.pandorabots.com/home.html) sendo capaz de responder cerca de 158 perguntas sobre diversos temas. A interface com o usuário é feita através de uma página web. MediBot [1] é um chatbot desenvolvido para fornecer informações sobre medicamentos e seus riscos. Ele foi implementado em favaScript utilizando NodefS, e pode ser utilizado através do aplicativo Telegram e interface web. HelpCare [10] é um chatbot que foi desenvolvido para auxiliar pacientes com doenças crônicas, como diabetes, colesterol alto e hipertensão arterial. Ele foi desenvolvido através da ferramenta de computação cognitiva IBMWatson.

\section{O PipaBot}

Nas próximas subseções nós apresentamos a metodologia, a arquitetura, as etapas e ferramentas usadas no desenvolvimento e a avaliação do PipaBot.

\subsection{Metodologia}

O chatbot vem sendo desenvolvido com base na metodologia Lean Inception [3]. O objetivo da metodologia Lean Inception [3] é desenvolver versões incrementais do Mínimo Produto Viável (MPV).

Para tanto, a metodologia usa o conceito Lean Startup [11] onde o MPV é o resultado principal do ciclo construir - medir aprender, ou seja, o MPV é o artefato a ser construído [3]. O PipaBot está na versão 3 e os detalhes do desenvolvimento são apresentados nas próximas subseções.

\subsection{Arquitetura do PipaBot}

A arquitetura do PipaBot é composta por um cliente, um gateway e servidores, como apresentado na Figura 1.

O cliente (Frontend) é o aplicativo final pelo qual o usuário envia e recebe as mensagens, como Facebook Messenger, Telegram, websites, entre outros. No caso do PipaBot, ele foi integrado ao Facebook Messenger e ao website do PIPA UFRJ.

O gateway é o serviço no qual ele se conecta e é responsável por encaminhar as mensagens para os servidores. No caso do Facebook Messenger, a mensagem é encaminhada para os servidores do Facebook que procura o endereço dos servidores responsáveis por tratá-la.

Os servidores são os responsáveis por tratar as mensagens recebidas, classificá-las em um fluxo de conversa, e enviar as respostas de volta para o gateway que, por sua vez, encaminha para o usuário.

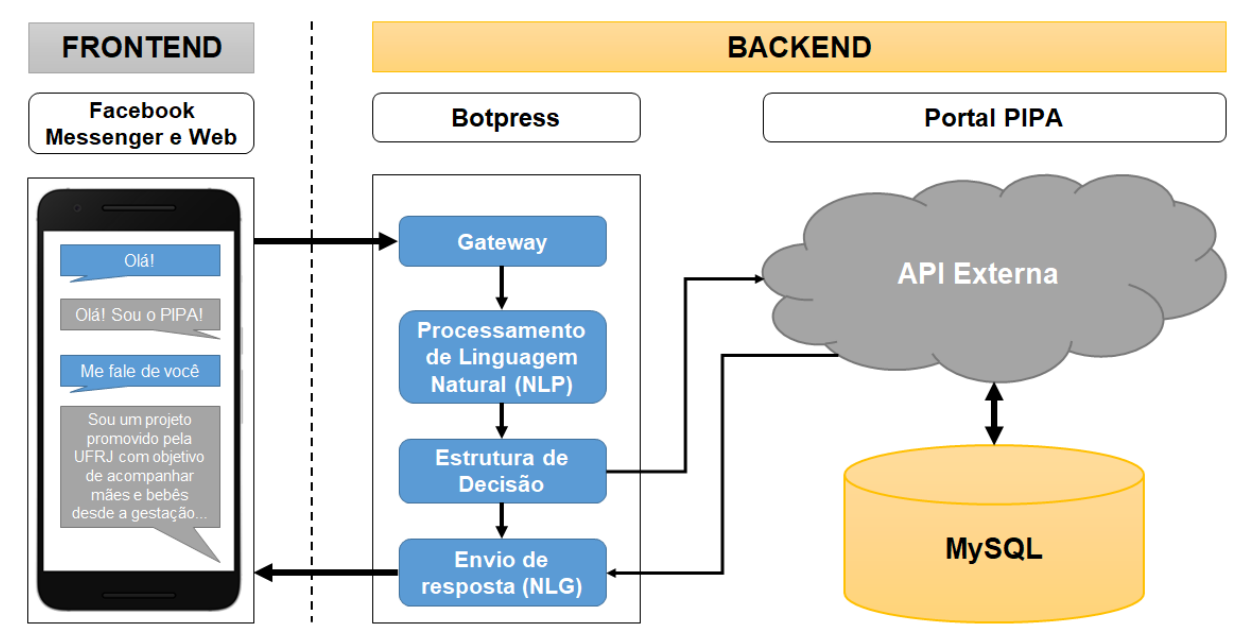

Figura 1: Arquitetura do PipaBot 


\subsection{Fluxo de conversa do PipaBot}

O fluxo de conversa é um conjunto de mensagens e interações menores que buscam alcançar um objetivo. A classificação em um fluxo de conversa é definida através de modelos de classificação de intenções, utilizando processamento de linguagem natural. O modelo é treinado com diversas frases para cada intenção, e sua saída é um vetor dos intents, com cada elemento contendo um número que vai de zero a um que determina a porcentagem de confiança na classificação para aquele elemento. $\mathrm{O}$ intent com o maior valor de confiança desse vetor é o utilizado para determinar o fluxo da conversa a ser seguida.

No caso do PipaBot, ao perguntarmos sobre o projeto, ele nos direciona para o fluxo de mensagens com as informações adequadas, como pode ser visto na Figura 2.

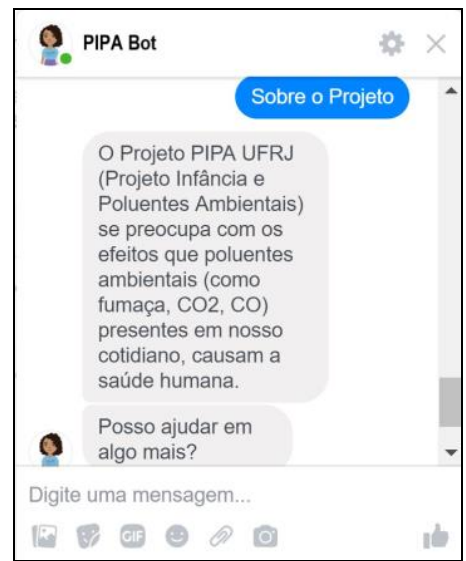

Figura 2: Diálogo com informações sobre o PIPA UFRJ

Quando questionado sobre o cadastro no canal, o PipaBot direciona o usuário para um fluxo de verificação de identidade. Ele verifica se o usuário já é um participante do PIPA UFRJ, como pode ser visto na Figura 3. Uma demonstração do PipaBot pode ser encontrada em: https://youtu.be/nybTVdPuJyw.

\subsection{Desenvolvimento}

Para apoiar o desenvolvimento do PipaBot, foi utilizado o framework open source Botpress. O objetivo desse framework é permitir a construção de chatbots através de interfaces de criação de fluxos simples. Ele é customizável de modo a permitir novas implementações, o uso de módulos desenvolvidos pela comunidade ou a criação do seu próprio módulo caso seja necessário.

O backend do framework é inteiramente desenvolvido em Javascript através do NodefS e suporta banco de dados SQLite e PostgreSQL de forma nativa. Sua interface de administração utiliza React, Redux e Bootstrap.

A escolha da plataforma do Facebook Messenger como canal de comunicação ocorreu porque o Facebook é a segunda rede social mais utilizada no Brasil (59\%), perdendo somente para o YouTube $(60 \%)^{1}$. O Facebook conta com cerca de 130 milhões de usuários ativos por mês e sua base de usuários vem crescendo cerca de $7 \%$ ao ano. Desses usuários, cerca de $92 \%$ acessam as redes sociais via aplicativos móveis. Por ser uma rede bastante difundida e tendo seus aplicativos instalados na maior parte dos celulares, entendemos que o Facebook é a rede social que mais pode aproximar os participantes do projeto.

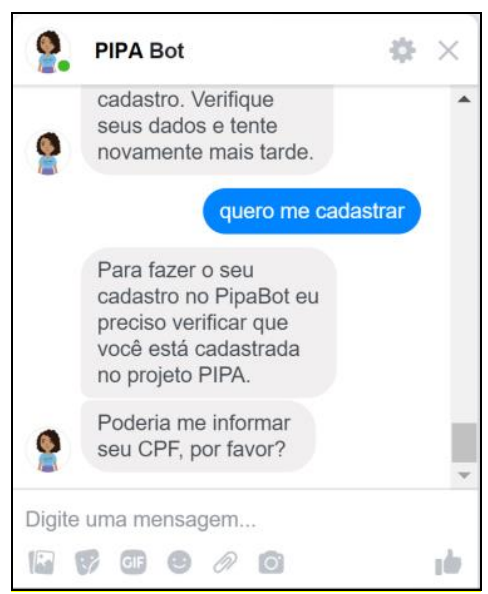

Figura 3: Diálogo com informações sobre o cadastro no PipaBot

Para integrar o Facebook Messenger ao Botpress foi necessária a instalação de um módulo de conexão para adicionar suporte ao canal. Também foi necessário criar um aplicativo na área de desenvolvedores do Facebook, que é por onde são solicitadas as permissões de acesso às informações dos usuários.

Determinados fluxos de conversa do PipaBot só podem ser acessados por participantes do PIPA UFRJ. Para que essa verificação fosse feita, foi necessário integrar o PipaBot ao Portal PIPA UFRJ, local onde todos os participantes do projeto estão cadastrados. Então, foi desenvolvido um plugin que estende a API do Portal, permitindo que sejam realizadas verificações sobre a existência de um participante no projeto, além de permitir o vínculo do PipaBot ao participante através de seus dados cadastrais.

\subsection{Avaliação}

A primeira versão do PipaBot (MPV 1) foi gerada após a primeira reunião com a equipe do projeto onde foram identificados os primeiros requisitos. Essa versão teve como objetivo mostrar o potencial de um chatbot para atrair e fidelizar os participantes do projeto. A segunda versão (MPV 2) foi gerada contemplando novas ações identificadas com o cliente. As duas primeiras versões foram testadas em laboratório e apresentadas ao cliente, gerando novas demandas e melhorias. A versão atual (MPV 3) do PipaBot contempla diferente ações, tais como: i) cadastro de uma

\footnotetext{
https://exame.abril.com.br/negocios/dino/62-da-populacao-brasileira-esta-ativanas-redes-sociais
} 
participante do projeto no PipaBot; ii) obtenção de informações sobre o projeto, consultas médicas, resultados de exames médicos, entre outros.

O MPV 3 do PipaBot foi avaliado segundo o modelo de aceitação de tecnologia (TAM - Tecnological Acceptance Model) [5]. Esse modelo tem como objetivo avaliar o impacto de uma nova tecnologia do ponto de vista do usuário. Para tanto, duas perspectivas são observadas: utilidade percebida e facilidade de uso percebida. A facilidade de uso foi caracterizada pelo número de mensagens trocadas com o chatbot e o tempo de resposta. A utilidade foi caracterizada pela pertinência e adequação do conteúdo da resposta.

A avaliação foi planejada de acordo com o paradigma GQM (Goal Question Metric - Objetivo Questão Métrica) [2] que é bem consolidado em Engenharia de Software. Para o modelo GQM dois objetivos $\left(\mathrm{O}_{1}\right.$ e $\left.\mathrm{O}_{2}\right)$ e seis questões $(\mathrm{Q} 1, \ldots, \mathrm{Q} 6)$ foram definidos como apresentado nas Tabelas 1 e 2.

Tabela 1: Objetivos

\begin{tabular}{|c|c|}
\hline \multicolumn{2}{|c|}{ Objetivo 1 $\left(\mathbf{O}_{1}\right)$} \\
\hline Analisar & O PipaBot \\
\hline Com o propósito de & Caracterizar \\
\hline Com respeito a & Facilidade de uso \\
\hline $\begin{array}{c}\text { Sob o ponto de vista } \\
\text { de }\end{array}$ & Profissionais de computação e saúde \\
\hline $\begin{array}{c}\text { No contexto de } \\
\text { Analisar }\end{array}$ & $\begin{array}{c}\text { Profissionais de computação e saúde } \\
\text { realizando diálogos com o PipaBot }\end{array}$ \\
\hline Objetivo 2 $\left(\mathbf{O}_{2}\right)$ \\
\hline Com o propósito de & O PipaBot \\
\hline Com respeito a & Utilidade \\
\hline $\begin{array}{c}\text { Sob o ponto de vista } \\
\text { de }\end{array}$ & Profissionais de computação e saúde \\
\hline No contexto de & $\begin{array}{c}\text { Profissionais de computação e saúde } \\
\text { realizando diálogos com o PipaBot }\end{array}$ \\
\hline
\end{tabular}

A avaliação foi feita por dois grupos distintos (13 participantes): profissionais de computação - que não fizeram parte do desenvolvimento do bot e do projeto de pesquisa - e de saúde (estudantes de graduação e pós-graduação). Os participantes receberam um roteiro de avaliação com cinco possíveis cenários (informações sobre o PIPA UFRJ, cadastro no portal do PIPA UFRJ, cadastro no PipaBot, informações sobre exames e informações sobre consultas médicas) a serem avaliados.

Após a execução dos cenários de diálogo com o PipaBot, os participantes responderam um questionário composto de questões objetivas (Tabela 2) com opções de resposta em uma escala Likert de quatro (Concordo totalmente, Concordo parcialmente, Discordo parcialmente e Discordo totalmente) ou cinco pontos (Concordo totalmente, Concordo parcialmente, Indiferente, Discordo parcialmente e Discordo totalmente).
Tabela 2: Questões

\begin{tabular}{|c|l|}
\hline Objetivos & \multicolumn{1}{c|}{ Questões } \\
\hline $\mathrm{O}_{1}$ & Q1 - O PipaBot é fácil de usar \\
\hline $\mathrm{O}_{1}$ & $\begin{array}{l}\text { Q2 - O PipaBot responde rapidamente aos meus } \\
\text { questionamentos }\end{array}$ \\
\hline $\mathrm{O}_{1}$ & $\begin{array}{l}\text { Q3- Eu posso obter a informação que desejo com } \\
\text { poucas perguntas }\end{array}$ \\
\hline $\mathrm{O}_{1}$ & $\begin{array}{l}\text { Q4- Os elementos de navegação (menus e botões) } \\
\text { do PipaBot facilitam a interação ao longo da } \\
\text { conversa }\end{array}$ \\
\hline $\mathrm{O}_{2}$ & $\begin{array}{l}\text { Q5- O PipaBot fornece respostas pertinentes aos } \\
\text { meus questionamentos }\end{array}$ \\
\hline $\mathrm{O}_{2}$ & $\begin{array}{l}\text { Q6 - O PipaBot fornece respostas adequadas aos } \\
\text { meus questionamentos }\end{array}$ \\
\hline
\end{tabular}

Além disso, para cada questão inserimos um campo de comentários (texto livre) para que os participantes pudessem expor suas ideias e incluir sugestões.

O grupo de profissionais de computação é caracterizado por: seis participantes do gênero masculino; sendo quatro alunos de graduação (66,7\%) e dois alunos de mestrado (33,3\%); com diferentes faixas etárias (cinco participantes entre 21 e 30 anos, e um partipante entre 31 e 40 anos). Os profissionais de saúde se caracterizam por: sete participantes do gênero feminino; sendo três alunas de graduação (42,9\%), três alunas de mestrado (42,9\%) e uma aluna de doutorado (14,3\%); com diferentes faixas etárias (uma participante com menos de 21 anos, cinco participantes entre 21 e 30 anos, e uma participante entre 41 e 50 anos). As Tabelas 3 e 4 apresentam os dados coletados através do questionário.

Tabela 3: Respostas dos Profissionais de Computação

\begin{tabular}{|c|c|c|c|c|c|c|c|}
\hline & Q1 & Q2 & Q3 & Q4 & Q5 & Q6 & Total \\
\hline $\begin{array}{c}\text { Concordo } \\
\text { totalmente }\end{array}$ & 1 & 6 & 4 & 5 & 1 & 2 & 19 \\
\hline $\begin{array}{c}\text { Concordo } \\
\text { parcialmente }\end{array}$ & 5 & 0 & 2 & 1 & 5 & 4 & 17 \\
\hline Indiferente & - & - & - & 0 & - & - & 0 \\
\hline $\begin{array}{c}\text { Discordo } \\
\text { parcialmente }\end{array}$ & 0 & 0 & 0 & 0 & 0 & 0 & 0 \\
\hline $\begin{array}{c}\text { Discordo } \\
\text { totalmente }\end{array}$ & 0 & 0 & 0 & 0 & 0 & 0 & 0 \\
\hline
\end{tabular}

Como pode ser observado na Tabela 3, para o grupo de profissionais de computação, todas as questões tiveram respostas no quadrante positivo ("concordo totalmente" ou "concordo parcialmente") da escala Likert. As questões Q1 e Q5 tiveram o maior índice (83\%) de respostas do tipo "concordo parcialmente". Os comentários dos participantes abordam que a estrutura do fluxo relacionado às consultas médicas não é de fácil entendimento para os usuários. Além disso, eles sugerem a integração do PipaBot com um sistema externo de agendamento eletrônico.

Na Tabela 4, para o grupo de profissionais da saúde, também podemos observar que todas as respostas ficaram no quadrante 
positivo. Entretanto, para esse grupo de usuários, podemos notar que em todas as questões a opção de resposta "concordo totalmente" teve um índice alto, somando $76 \%$ do total de todas as respostas do questionário.

Tabela 4: Respostas dos Profissionais de Saúde

\begin{tabular}{|c|c|c|c|c|c|c|c|}
\hline & Q1 & Q2 & Q3 & Q4 & Q5 & Q6 & Total \\
\hline $\begin{array}{c}\text { Concordo } \\
\text { totalmente }\end{array}$ & 6 & 5 & 5 & 7 & 4 & 5 & 32 \\
\hline $\begin{array}{c}\text { Concordo } \\
\text { parcialmente }\end{array}$ & 1 & 2 & 2 & 0 & 3 & 2 & 10 \\
\hline Indiferente & - & - & - & 0 & - & - & 0 \\
\hline $\begin{array}{c}\text { Discordo } \\
\text { parcialmente }\end{array}$ & 0 & 0 & 0 & 0 & 0 & 0 & 0 \\
\hline $\begin{array}{c}\text { Discordo } \\
\text { totalmente }\end{array}$ & 0 & 0 & 0 & 0 & 0 & 0 & 0 \\
\hline
\end{tabular}

Em geral, os comentários do grupo de profissionais da saúde foram: sugestão para adicionar novas informações (por exemplo, o que é a Maternidade Escola da UFRJ) na base de conhecimento do PipaBot; sugestão para implementar novos fluxos de conversa ou novas opções para os fluxos existentes (por exemplo, local onde acontecem as atividades do PIPA UFRJ).

O grupo de profissionais de saúde que teve o maior indície de respostas "concordo totalmente" pode representar uma ameaça à validade do estudo. Este grupo carece de tecnologias e possívelmente as participantes mostraram a expectativa positiva de ter uma tecnologia. Para minimizar este risco, a avaliação também foi executada com profissionais de computação, que por sua vez apresentam uma expectativa um pouco menos positiva. Além disso, nós planejamos realizar uma nova avaliação com outros grupos.

De modo geral, os cenários propostos para a avaliação do chatbot eram de fácil entendimento e possivelmente não geraram frustações. Este ponto também pode ser visto como uma ameaça à validade do estudo. Futuramente nós pretendemos implementar novas ações no chatbot segundo as sugestões recebidas, e realizar um novo estudo de avaliação da tecnologia.

Contudo, quando nós analisamos de forma conjunta as respostas dos 13 participantes podemos considerar que os resultados são satisfatórios. Porém não podemos generalizar os resultados.

\section{Considerações finais e trabalhos futuros}

Neste artigo foi apresentado o PipaBot, o chatbot do PIPA UFRJ, que tem como objetivo fomentar um canal de comunicação entre as participantes (as mães) e os responsáveis do projeto.

A versão atual do Pipabot (MPV 3) permite que o usuário obtenha informações sobre o PIPA UFRJ, faça o seu cadastro no PipaBot, faça questionamentos sobre consultas médicas, resultados de exames médicos, entre outros. O chatbot foi avaliado segundo o modelo de aceitação da tecnologia (TAM Tecnological Acceptance Model) e os resultados iniciais foram satisfatórios do ponto de vista técnico.
O PipaBot foi desenvolvido a partir de uma necessidade do projeto PIPA UFRJ, o qual tem a expectativa de atender em torno de 2000 participantes das iniciativas em prol da saúde gestacional e infantil oferecidas. Como trabalho futuro, nós planejamos uma avaliação do PipaBot com os usuários finais (mães e gestantes do PIPA UFRJ) e disponibilização do chatbot em outras plataformas de mensagem instantânea, como WhatsApp. Além de prover a integração de novas ações alinhadas com as ações atualmente realizadas no PIPA UFRJ, tais como: agendamento e lembrete de consultas/exames; envio de mensagens proativas e periódicas para os usuários.

\section{AGRADECIMENTOS}

Os autores agradecem as pessoas que participaram do estudo de avaliação do PipaBot. Lucas agradece o PIPA UFRJ pela bolsa PIBIC. Taisa agradece o CNPq pela bolsa de pós-doutorado (154004/2018-9). Prof. Guilherme Travassos é pesquisador CNPq (304234/2018-4). Os autores agradecem aos membros da equipe do PIPA UFRJ pelo apoio e sugestões.

\section{REFERENCES}

[1] C.V.S. Avila, T.V. Rolim, J.W.F. da Silva, and V.M.P. Vidal. 2019. MediBot: Um chatbot para consulta de riscos e informações sobre medicamentos. In Anais Estendidos do XIX SBCAS - Workshop de Ferramentas e Aplicações, 16.

[2] V. R. Basili, C. Caldiera, and H. D. Rombach. 1994. Encyclopedia of Software Engineering. In Goal Question Metric Paradigm. John Wiley \& Son, 528-532.

[3] Paulo Caroli. 2017. To the point: a recipe for creating lean products. Retrieved from http://leanpub.com/tothepoint

[4] Kedar S. Chikorde and Pankaja B. Patil. 2017. Natural Language Processing based chatbot for IoT dashboards. International fournal of current Engineering and Scientific Research 4, 8 (2017), 87-90.

[5] Fred D. Davis. 1993. User acceptance of information technology: system characteristics, user perceptions and behavioral impacts. International fournal of Man-Machine Studies 38, 3 (1993), 475-487. DOI:https://doi.org/10.1006/imms.1993.1022

[6] Charbel El Kaed, Andre Ponnouradjane, and Dhaval Shah. 2018. A Semantic Based Multi-Platform IoT Integration Approach from Sensors to Chatbots. In Proceedings of the 2018 Global Internet of Things Summit (GIoTS), 1-6. DOI:https://doi.org/10.1109/GIOTS.2018.8534520

[7] Matheus Fossatti, Roberto Rabello, and Ana Carolina De Marchi. 2011. AGEbot: Um chatterbot em AIML voltado para responder questões sobre Epilepsia. In Anais do XI Workshop de Informática Médica, 10.

[8] Rohan Kar and Rishin Haldar. 2016. Applying Chatbots to the Internet of Things: Opportunities and Architectural Elements. International fournal of Advanced Computer Science and Applications 7, 11 (2016). DOI:https://doi.org/10.14569/IJACSA.2016.071119

[9] Yang Lu. 2019. Artificial intelligence: a survey on evolution, models, applications and future trends. Fournal of Management Analytics 6, 1 (2019), 1-29. DOI:https://doi.org/10.1080/23270012.2019.1570365

[10] N. Oliveira, A. Costa, D. Araujo, and C. Portela. 2019. HelpCare: Um Protótipo de ChatBot para o Auxílio do Tratamento de Doenças Crônicas. In Anais Estendidos do XIX SBCAS - Workshop de Ferramentas e Aplicações, 282-287.

[11] Eric Ries. 2011. The Lean Startup: How Today's Entrepreneurs Use Continuous Innovation to Create Radically Successful Businesses. Crown Business.

[12] Richard S. Wallace. 2009. The Anatomy of A.L.I.C.E. In Parsing the Turing Test, Robert Epstein, Gary Roberts and Grace Beber (eds.). Springer Netherlands, Dordrecht, 181-210. DOI:https://doi.org/10.1007/978-1-40206710-5_13

[13] Joseph Weizenbaum. 1966. ELIZA-a computer program for the study of natural language communication between man and machine. Communications of the ACM 9, 1 (1966), 36-45. DOI:https://doi.org/10.1145/365153.365168 